\title{
Duration of simulated microgravity affects the differentiation of mesenchymal stem cells
}

\author{
LI XUE ${ }^{1 *}$, YAOHUI $\mathrm{LI}^{2 *}$ and JUN CHEN ${ }^{3}$ \\ ${ }^{1}$ Department of Urology, The Second Affiliated Hospital, Xi'an Jiaotong University; Departments of ${ }^{2}$ Pneumology \\ and ${ }^{3}$ Encephalopathy, Traditional Chinese Medicine Hospital of Shaanxi, Xi'an, Shaanxi 710014, P.R. China
}

Received January 5, 2016; Accepted February 9, 2017

DOI: $10.3892 / \mathrm{mmr} .2017 .6357$

\begin{abstract}
Previous evidence has suggested that physical microenvironments and mechanical stresses, independent of soluble factors, influence mesenchymal stem cell (MSC) fate. In the present study, simulated microgravity (SMG) was demonstrated to regulate the differentiation of mesenchymal stem cells. This may be a novel strategy for tissue engineering and regenerative medicine. Rat MSCs were cultured for $72 \mathrm{~h}$ or 10 days in either normal gravity or a clinostat to model microgravity, followed with culture in diverse differential media. A short period of stimulation (72 h) promoted MSCs to undergo endothelial, neuronal and adipogenic differentiation. In comparison, extended microgravity (10 days) promoted MSCs to differentiate into osteoblasts. A short period of exposure to SMG significantly decreased ras homolog family member A (RhoA) activity. However, RhoA activity significantly increased following prolonged exposure to SMG. When RhoA activity was inhibited, the effects of prolonged exposure to SMG were reversed. These results demonstrated that the duration of SMG regulates the differentiation fate of MSCs via the RhoA-associated pathway.
\end{abstract}

Correspondence to: Dr Jun Chen, Department of Encephalopathy, Traditional Chinese Medicine Hospital of Shaanxi Province, $2 \mathrm{Xi}$ Huamen Street, Xi'an, Shaanxi 710014, P.R. China

E-mail: chj2002819@163.com

*Contributed equally

Abbreviations: SMG, simulated microgravity; NG, normal gravity; MSCs, mesenchymal stem cells; ROCK, Rho-associated, coiled-coil-containing protein kinase; $\mathrm{mDia}$, mammalian diaphanous related protein; PPAR $\gamma 2$, peroxisome proliferator-activated receptor $\gamma 2$; MAP-2, microtubule-associated protein 2; Vwf, von Willebrand factor; ALP, alkaline phosphatase

Key words: microgravity, regeneration, cytoskeletal tension, differentiation

\section{Introduction}

Tissue engineering is an interdisciplinary field that focuses on developing biological substitutes that restore, maintain or improve natural tissue function (1). However, tissues are sophisticated structures formed by multiple cells types acting in concert under regulated conditions. In this context, one of the major difficulties in tissue engineering is acquiring sufficient numbers of cells. For this reason, researchers have focused on improving the differentiation potential of stem cells to accelerate organ regeneration.

Mechanical stimulation affects stem cell differentiation. Exposing cells to real or simulated microgravity (SMG) is a suitable technique for tissue engineering, as microgravity triggers cells to assemble three-dimensionally in vitro as they do in vivo (2-4). Human mesenchymal stem cells (hMSCs) cultured in SMG possess the proliferative characteristics of stem cells and retain their ability to differentiate into hyaline cartilage following transplantation (5). Huang et al (6) reported that microgravity induced MSCs to differentiate into adipocytes, which are force-insensitive cells. Chen et al (7) demonstrated that MSCs more readily differentiated into neuronal cells under SMG. However, modeled microgravity inhibited the differentiation of MSCs into osteoblasts (8). The functional mechanisms underlying the effect of SMG on MSC differentiation remains unclear.

Cytoskeletal tension and ras homolog family member A (RhoA) have previously been demonstrated to mediate the lineage decision of hMSCs (9). Increased actomyosin contractility promotes osteogenesis, while cytoskeleton disruption enhances adipogenesis (10). During spaceflight and under SMG conditions, cytoskeletal alterations occur in several cell types including lymphocytes, glial cells and stem cells (11-13). In addition, previous research demonstrated that MSCs are sensitive to gravity changes (6). MSC microtubules and $\alpha$-actin undergo remodeling following SMG stimulation (7). The Rho subfamily of Ras-related small GTPases is characterized predominantly by its regulation of the actin cytoskeleton. RhoA primarily mediates stress fiber formation (14). Thus, the present study examined whether SMG remodels the cytoskeleton by regulating the activity of RhoA. The purpose of the present study was to measure the effect of time-dependent changes in the cytoskeleton of MSCs on MSC differentiation following different conditions of modeled microgravity. 


\section{Materials and methods}

Preparation and culture of adult rat (r)MSCs. A total of 10 male Wistar rats (two weeks old) were purchased from the animal research center of the Fourth Military Medical University (Xi'an, China). The rats were housed in isolated and ventilated cages, and the animal protocol was approved by the Fourth Military Medical University Medical Ethics Committee (Xi'an, Shaanxi, China; approval no. XJYYLL-2015508). The animals were acclimated to the laboratory environment for 5-7 days before use. While in their home cage environment, the animals were allowed access to a standard animal diet and tap water ad libitum. The room was maintained at $20-23^{\circ} \mathrm{C}$ with a $12 \mathrm{~h}: 12 \mathrm{~h}$ light:dark cycle. The animals were deeply anesthetized using inhaled isoflurane (1-3\% or as required) before all surgery. All efforts were made to minimize animal suffering and to reduce the number of animals used. rMSCs were collected as previously described by Azizi et al (15), with slight modifications. The Wistar rats were euthanized and bilateral femurs and tibias were removed. Mesenchymal stem cells were cultured in Dulbecco's modified Eagle's medium (DMEM; Gibco; Thermo Fisher Scientific, Inc., Waltham, MA, USA) supplemented with $10 \%$ fetal bovine serum (FBS; Gibco; Thermo Fisher Scientific, Inc.), $100 \mathrm{U} / \mathrm{ml}$ penicillin $\mathrm{G}$ and $100 \mathrm{mg} / \mathrm{ml}$ streptomycin (Gibco; Thermo Fisher Scientific, Inc.) at $2.5 \times 10^{5} / \mathrm{cm}^{2}$. Fluorescence phase-contrast microscopy (Axiocam MR R3; Carl Zeiss AG, Oberkochen, Germany) was used to observe rMSCs every 2-3 days. Fluorescence activated cell sorting (FACS) analysis of CD44, CD34, CD90 and CD45 was performed in rMSCs.

Clinorotation to modeled microgravity. The weightless environment from a clinostat, or rotating vessel, is regarded as 'modeled microgravity' (16). The conditions prevent the cell from being affected by gravity. The method is based on the hypothesis that sensing no gravity has similar effects to being weightless. The clinostat model system (clinorotation) used in the present study provides a vector-averaged reduction in the apparent gravity on cell culture (Fig. 1A). Stem cells were seeded at a density of $1 \times 10^{5}$ cells on $2.5 \times 2.5 \mathrm{~cm}$ coverslips. The coverslips were inserted into the fixture of the chambers, filled completely with DMEM $+10 \%$ FBS and aspirated to eliminate air bubbles. The chambers were divided into two groups randomly: Normal gravity (NG) controls without rotation for $72 \mathrm{~h}$ and SMG groups with clinorotation $(30 \mathrm{xg})$ for $72 \mathrm{~h}$ or 10 days.

Neuronal differentiation. Following SMG stimulation, rMSCs were induced to differentiate as previously described, with modifications (17).Cellsweremaintained in pre-induction media consisting of DMEM, $0.1 \mathrm{mM} / 1$ 2-mercaptoethanol (Gibco; Thermo Fisher Scientific, Inc.) and 2\% dimethylsulfoxide (WAK-Chemie Medical GmbH, Steinbach, Germany) for $5 \mathrm{~h}$. Pre-induction media were removed, cells were transferred to neuronal induction media composed of DMEM $+10 \%$ FBS, $10 \mu \mathrm{g} / 1$ basic fibroblast growth factor (R\&D Systems, Inc., Minneapolis, MN, USA), $10 \mu \mathrm{g} / 1$ human epidermal growth factor (R\&D Systems, Inc.), $1 \mathrm{mM}$ dibutyrylcyclicn AMP (Sigma-Aldrich; Merck KGaA, Darmstadt, Germany) and 0.5 mM 3-isobutyl-1-methyl-xanthine (IBMX; Sigma-Aldrich; Merck KGaA) for 10 days.
Osteogenic differentiation. Induced osteogenic differentiation of MSCs was performed according to the accepted protocol of culturing cells in DMEM-advanced medium containing $10^{-8} \mathrm{M}$ dexamethasone, $10 \mathrm{mM}$ beta-glycerol 2-phosphate and $0.2 \mathrm{mM}$ 2-phospho-L-ascorbic acid (Sigma-Aldrich; Merck $\mathrm{KGaA})(18)$.

Endothelial differentiation. Endothelial differentiation was performed as described previously with modifications (19). Cells were incubated for up to 10 days in endothelial differentiation medium containing low glucose DMEM supplemented with $100 \mathrm{ng} / \mathrm{ml}$ vascular endothelial growth factor (Invitrogen; Thermo Fisher Scientific, Inc.), $50 \mathrm{ng} / \mathrm{ml}$ epidermal growth factor (Invitrogen; Thermo Fisher Scientific, Inc.), $1 \mu \mathrm{g} / \mathrm{ml}$ hydrocortisone (Sigma-Aldrich; Merck KGaA), 5\% FBS (Invitrogen; Thermo Fisher Scientific, Inc.), $100 \mathrm{U} / \mathrm{ml}$ penicillin and $100 \mathrm{mg} / \mathrm{ml}$ streptomycin.

Adipogenic differentiation of MSCs. Following culture under simulated microgravity for $72 \mathrm{~h}$ or 10 days, cells were cultured in adipogenic medium with $1 \mu \mathrm{M}$ dexamethasone, $10 \mu \mathrm{g} / \mathrm{ml}$ insulin, $500 \mu \mathrm{M}$ IBMX and $200 \mu \mathrm{M}$ indomethacin (20).

Fluorescence immunocytochemistry. Following SMG stimulation, the cytoskeletons of MSCs were observed. Cultured cells were fixed with $4 \%$ paraformaldehyde for 20 min, immersed in PBS for 10 min then exposed to $0.01 \%$ Triton X-100 at room temperature for $10 \mathrm{~min}$. Following rotation for $72 \mathrm{~h}$ or 10 days, rMSCs were blocked with $1 \%$ FBS at room temperature for $30 \mathrm{~min}$ then incubated with Texas Red-labeled phalloidin (T7471; 1:200; Invitrogen; Thermo Fisher Scientific, Inc.) and $\beta$-tubulin (SC398937; 1:200; Santa Cruz Biotechnology, Inc., Dallas, TX, USA) antibodies at $37^{\circ} \mathrm{C}$ for $1 \mathrm{~h}$. MSCs were stained with 4'6'-diamidino-2-phenylindole (1:500; Sigma-Aldrich; Merck KGaA) for 15 min for nuclear staining. To further examine the involvement of RhoA activation in the differentiation potential of rMSCs cultured in SMG, Y27632 (040012; Stemgent, Cambridge, MA, USA) was used to specifically block RhoA/rock signaling. Cells were pretreated with $20 \mu \mathrm{ml}$ inhibitor and stimulated with SMG for 10 days. ALP (LS-C44264; 1:200; LifeSpan BioSciences, Inc., Seattle, WA, USA) antibody was incubated as above. Cells were observed on a laser scanning confocal microscope (Leica Microsystems GmbH, Wetzlar, Germany) and imaged using a SPOT II camera (Diagnostic Instruments, Inc., Sterling Heights, MI, USA).

Reverse transcription polymerase chain reaction (RT-PCR) analysis. Total RNA was extracted from cultured cells in induction media using TRIzol reagent (Invitrogen; Thermo Fisher Scientific, Inc.), according to the manufacturer's protocol. The primer sequences used for microtubule-associated protein 2 (MAP-2), von Willebrand factor (vWF), alkaline phosphatase (ALP), peroxisome proliferator-activated receptor $\gamma 2$ (PPAR $\gamma-2)$ and GAPDH are presented in Table I and were synthesized by Beijing SBS Genetech Co., Ltd. (Beijing, China). RT-PCR using the Access RT-PCR reagent (Promega Corporation, Madison, WI, USA) was performed for 35 cycles, with each cycle consisting of $94^{\circ} \mathrm{C}$ for $30 \mathrm{sec}, 55^{\circ} \mathrm{C}$ for $30 \mathrm{sec}$ and $68^{\circ} \mathrm{C}$ for $1 \mathrm{~min}$. Samples $(10 \mu \mathrm{l})$ of each PCR 
product were size-fractionated by $1.5 \%$ agarose gel electrophoresis and the bands were visualized with ImageMaster VDS (Pharmacia Biotech; GE Healthcare Life Sciences, Chalfont, UK). The densitometry values were normalized to GAPDH and performed by Total Lab computer program version 1.11 (GE Healthcare Life Sciences, Chalfont, UK).

Oil red o staining. Oil red O kit (ab150678; Abcam, Cambridge, UK) was used to investigate the efficiency of adipogenic induction. Cells were fixed in $10 \%$ formalin for $10 \mathrm{~min}$, rinsed 3 times in $1 \mathrm{X}$ PBS, then placed in oil red $\mathrm{O}$ solution for $10 \mathrm{~min}$, rinsed in tap water and counterstained with hematoxylin for $1 \mathrm{~min}$. An inverted phase-contrast microscope (Eclipse TE 300; Nikon Co., Tokyo, Japan) was used for examination.

FACS. Following induction for 10 days, the differentiated cells were analyzed using FACS. The harvested cells washed in Dulbecco's PBS and incubated at $4^{\circ} \mathrm{C}$ for $30 \mathrm{~min}$ with antibodies for MAP-2 (13-1500; 1:200; Invitrogen; Thermo Fisher Scientific, Inc.), vWF (MA5-14029; 1:200; Invitrogen; Thermo Fisher Scientific, Inc.), ALP (LS-C44264; 1:200; LifeSpan BioSciences, Inc.) and PPAR $\gamma-2$ (PA3-821A; 1:200; Invitrogen; Thermo Fisher Scientific, Inc.) following blocking with normal goat serum $(10 \%)$ at $4^{\circ} \mathrm{C}$ for $30 \mathrm{~min}$. Cells were resuspended in DMEM/PBS containing a working dilution (1:100) of phycoerythrin-labeled goat anti-mouse immunoglobulin $\mathrm{G}$ (43R-1613; Fitzgerald, Acton, MA, USA) and incubated at $4^{\circ} \mathrm{C}$ for $30 \mathrm{~min}$. Cells were analyzed using a fluorescence activated cell sorter (FACScan) cytometer (Cytoflex S; Beckman Coulter Inc., Brea, CA, USA) with FlowJo software version 7.6 (Tree Star Inc, Ashland, OR, USA).

RhoA activity assay. The RhoA activity assay was performed according to the manufacturer's protocol (RhoA Pulldown Activation Assay kit; BK036; Cytoskeleton, Inc., Denver, CO, USA). Activated (GTP-bound) RhoA was pulled-down from whole cell lysates (300 $\mu \mathrm{g}$ protein/sample) with rhotekin-conjugated agarose beads for $1 \mathrm{~h}$ at $4^{\circ} \mathrm{C}$. The beads were collected by centrifugation for $1 \mathrm{~min}$ at $5,000 \mathrm{x} \mathrm{g}$ at $4^{\circ} \mathrm{C}$ and washed with lysis buffer, followed by wash buffer (1X lysis buffer). Activated RhoA was detached from the beads by boiling for 5 min in Laemmli reducing buffer (1X) immediately prior to separation by $12.5 \%$ SDS-PAGE, following which it was transferred to a polyvinylidene membrane. Following blocking for $1 \mathrm{~h}$ at room temperature in TBST containing 5\% non-fat dry milk, the membranes were incubated with a primary antibody directed against RhoA (21009; 1:200; Cytoskeleton, Inc.) overnight at $4^{\circ} \mathrm{C}$. Signals were detected using a horseradish peroxidase-conjugated goat anti-mouse immunoglobulin G1 secary antibody incubated for $1 \mathrm{~h}$ at room temperature (R-05071-500; 1:1,000; Advansta, Menlo Park, CA, USA) and an enhanced chemiluminescence detection system (GE Healthcare Life Sciences). The optical densities were analyzed by using ImageMaster 2D Platinum version 5.0 (GE Healthcare Life Sciences).

Statistical analysis. Statistical analysis was carried out using SPSS version 10.0 for Window software (SPSS, Chicago, IL, USA). Statistical analysis was performed using the Student's t-test on pixel intensity data generated from scanning autoradiographs. $\mathrm{P}<0.05$ was considered to indicate a statistically significant difference. All experiments were repeated at least three times. Data were expressed as the mean \pm standard error of the mean.

\section{Results}

Characterization of cultured rMSCs. Flow cytometry analysis was performed on cells at passage 2. Undifferentiated rMSCs expressed CD90 and CD44, with a positive ratio of 97.37 and $94.35 \%$, respectively. These cells were negative for CD45 and CD34 (Fig. 1B). Following 2 passages, adherent cells exhibited spindle or large flattened morphology, and following SMG treatment the shape of the cells visibly shifted from spindle to round (Fig. 1C). This morphological change may reflect cytoskeletal reorganization; therefore, the cytoskeletal change following SMG intervention was analyzed at different time points.

Cytoskeletal reorganization following SMG stimulation. In the present study, MSC microtubules were highly sensitive to weightlessness. Modified microtubules were observed in some MSC clusters under SMG, and few fibers were distinguishable against the background under SMG for $72 \mathrm{~h}$ (Fig. 1D). However, following 10 days of SMG culture, cells appeared to have reestablished more microtubules (Fig. 1D). Compared with the static control, $\alpha$-actin filaments underwent remodeling following SMG stimulation, appearing diffuse following $72 \mathrm{~h}$ rotation (Fig. 1E). However, following rotation for 10 days, re-concentrated actin was observed (Fig. 1E).

Neuronal differentiation. MSCs were cultured using a clinostat to simulate microgravity conditions for $0 \mathrm{~h}(\mathrm{NG}), 72 \mathrm{~h}$ or 10 days. Following SMG, cells were incubated for up to 10 days in differentiation medium. At day 10, cells were lysed with TRIzol for total RNA extraction. The expression of the neuron-specific marker MAP-2 was analyzed by semi-quantitative RT-PCR. The results revealed that expression of MAP-2 increased significantly following $72 \mathrm{~h}$ SMG compared with the NG group $(\mathrm{P}<0.05$; Fig. 2A). In contrast, expression significantly decreased following 10 days SMG stimulation compared with the $72 \mathrm{~h}$ group $(\mathrm{P}<0.05$; Fig. $2 \mathrm{~A})$.

Osteogenic differentiation. mRNA expression levels of ALP, a marker of osteogenesis, were investigated. The results revealed that ALP expression was reduced significantly following $72 \mathrm{~h}$ exposure to $\mathrm{SMG}$ compared with the $\mathrm{NG}$ group $(\mathrm{P}<0.05$; Fig. 2B). ALP mRNA expression levels significantly increased following 10 day SMG exposure compared with $72 \mathrm{~h} \mathrm{SMG}$ $(\mathrm{P}<0.05$; Fig. 2B)

Endothelial differentiation. rBMSCs were cultured under SMG conditions for $72 \mathrm{~h}$ or 10 days, then further cultured for 10 days in differential medium. The expression level of the endothelium- specific marker vWF was analyzed by semi-quantitative RT-PCR. vWF mRNA expression levels were significantly higher in the $72 \mathrm{~h}$ group compared with the $\mathrm{NG}$ group, and four-fold higher in the $72 \mathrm{~h}$ group compared with 10 day group (both $\mathrm{P}<0.05$; Fig. 2C). 
Table I. Primers used in the semi-quantitative reverse transcription-polymerase chain reaction.

\begin{tabular}{|c|c|c|}
\hline Primer & Gene sequence $\left(5^{\prime}-3^{\prime}\right)$ & Length (bp) \\
\hline MAP-2 & $\begin{array}{l}\text { Forward TTGAAGGTTAAAATGCATCTGA } \\
\text { Reverse GGCATTTCAAGGAAAAACTCA }\end{array}$ & 152 \\
\hline vWF & $\begin{array}{l}\text { Forward CACCCAAGTTCCACATCAGC } \\
\text { Reverse TCCACCATCGTCTGCTTCAT }\end{array}$ & 120 \\
\hline ALP & $\begin{array}{l}\text { Forward GGAAGGGTCAGTCAGGTT } \\
\text { Reverse GTGGGCCGCTCTAGGCACCAA }\end{array}$ & 366 \\
\hline PPAR $\gamma 2$ & $\begin{array}{l}\text { Forward 5'-TTGATTTCTCCAGCATTTC-3' } \\
\text { Reverse GCTCTACTTTGATCGCACT }\end{array}$ & 360 \\
\hline GAPDH & $\begin{array}{l}\text { Forward 5'-CCACAGTCCATGCCATCAC-3' } \\
\text { Reverse TCCACCACCCTGTTGCTGTA }\end{array}$ & 452 \\
\hline
\end{tabular}

MAP-2, microtubule-associated protein 2; vWF, von Willebrand factor; ALP, alkaline phosphatase; PPAR $\gamma 2$, peroxisome proliferator-activated receptor $\gamma 2$; GAPDH, glyceraldehyde 3-phosphate dehydrogenase.
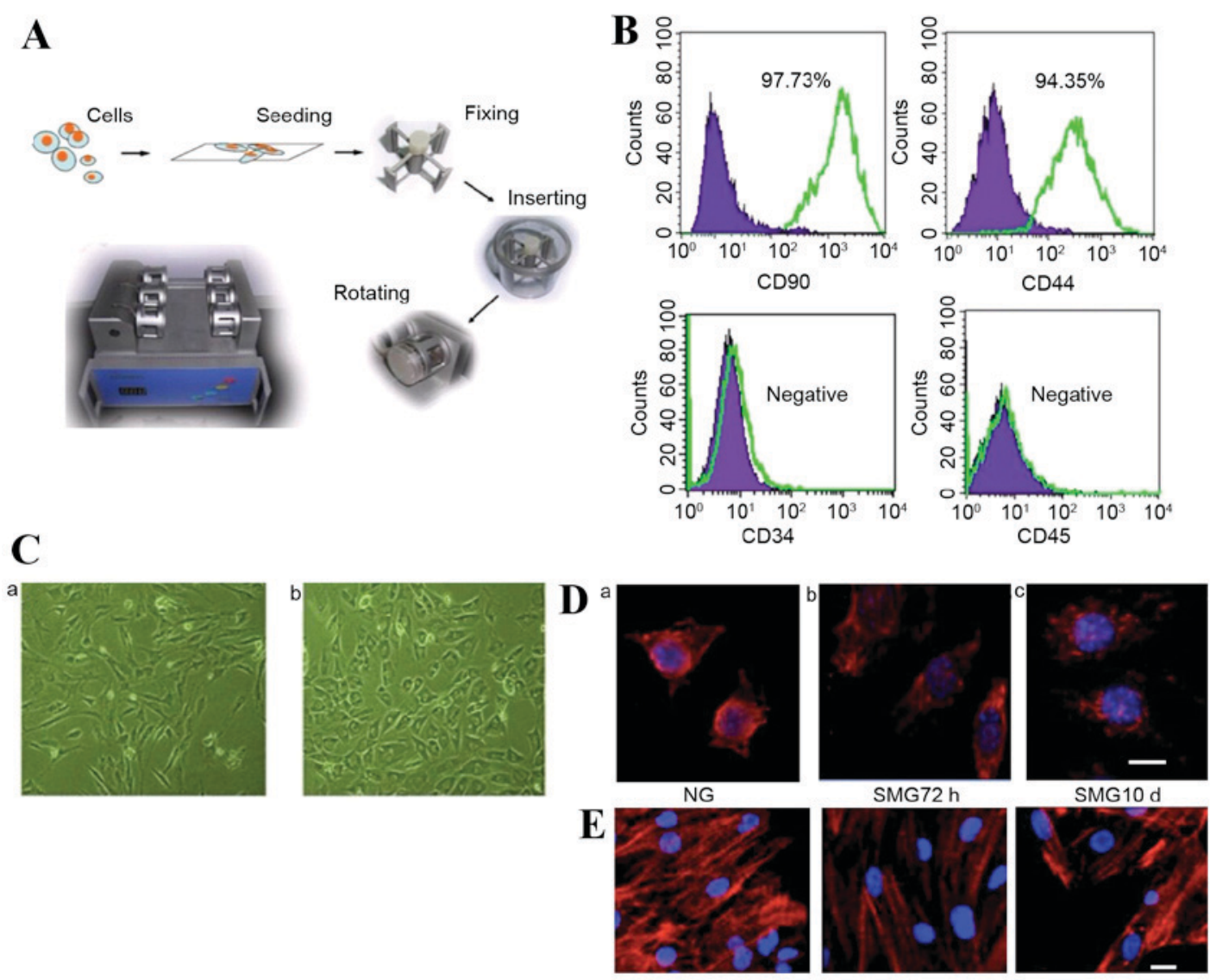

Figure 1. (A) The SMG clinostat model system. (B) Fluorescence-activated cell sorting analysis of CD44, CD34, CD90 and CD45 in rMSCs. (C) Light microscopy analysis of the shape change of rMSCs under (a) NG and (b) SMG conditions (x50 magnification). (D) Microtubule formation was disrupted following SMG. Scale bar=10 $\mu \mathrm{m}$. Modified microtubules were observed in rMSCs compared to (a) the NG control following (b) $72 \mathrm{~h} \mathrm{SMG,} \mathrm{but} \mathrm{cells} \mathrm{appeared} \mathrm{to} \mathrm{have}$ reestablished microtubules following (c) 10 days SMG. (E) $\alpha$-actin filaments underwent remodeling following SMG stimulation, appearing diffuse compared to (a) the NG control following (b) $72 \mathrm{~h} \mathrm{SMG.} \mathrm{However,} \mathrm{following} \mathrm{(c)} 10$ day SMG, re-concentrated actin was observed. SMG, simulated microgravity; rMSCs, rat mesenchymal stem cells; NG, normal gravity.

Adipogenic differentiation. PPAR $\gamma-2$ is a critical transcription factor involved in adipogenic differentiation in MSCs. To measure the potential of differentiation into adipocytes, PPAR $\gamma-2$ mRNA expression levels were measured by semi-quantitative RT-PCR in MSCs under simulated microgravity. The results revealed that
mRNA expression levels of PPAR $\gamma-2$ increased significantly following $72 \mathrm{~h}$ SMG compared with the NG group $(\mathrm{P}<0.05$; Fig. 2D). Notably, following long term stimulation for 10 days, PPAR $\gamma-2$ mRNA expression levels significantly decreased by $\sim 60 \%$ compared with $72 \mathrm{~h}$ group $(\mathrm{P}<0.05$; Fig. $2 \mathrm{D})$. 

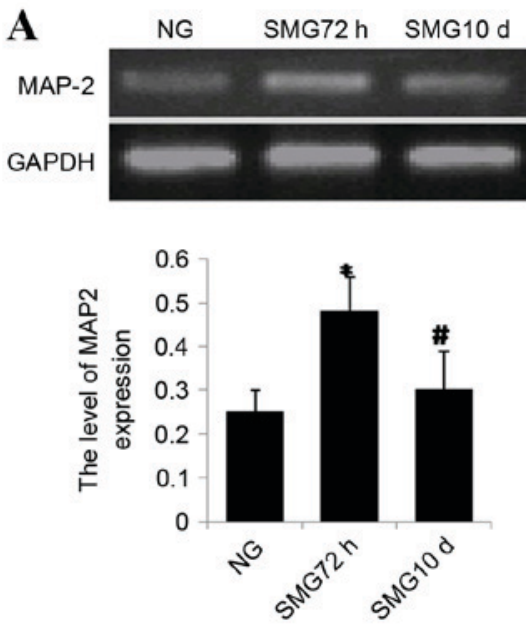

C



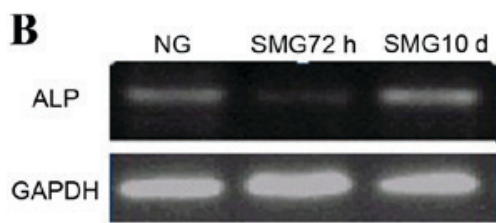
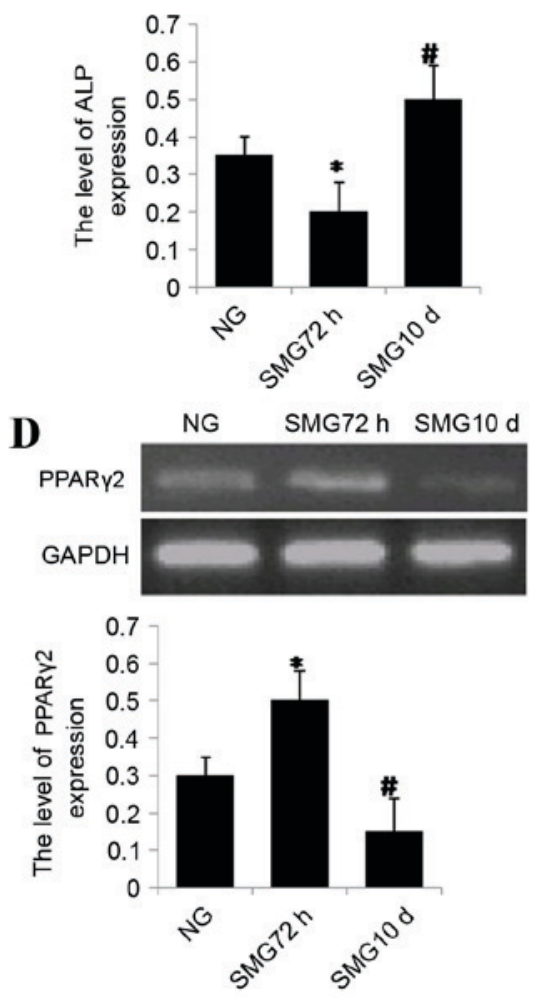

Figure 2. (A) Semi quantitative reverse transcription polymerase chain reaction analysis of (A) MAP-2 following neuronal differentiation, (B) ALP following osteogenic differentiation, (C) vWF following endothelial differentiation and (D) PPAR $\gamma 2$ expression following adipogenic differentiation. Gene expression was normalized to endogenous GAPDH. The results represent three independent experiments. ${ }^{*} \mathrm{P}<0.05$ vs. NG group, ${ }^{\sharp} \mathrm{P}<0.05$ vs. $72 \mathrm{~h}$. MAP-2, microtubule-associated protein 2; ALP, alkaline phosphatase; vWF, von Willebrand factor; PPAR $\gamma 2$, peroxisome proliferator-activated receptor $\gamma 2$; GAPDH, glyceraldehyde 3-phosphate dehydrogenase; NG, normal gravity; SMG, simulated microgravity.

Confirmation of differentiation by FACS. The FACS results revealed that MAP-2-positive cells from the SMG $72 \mathrm{~h}$ group accounted for $66.3 \%$ of the cells, compared with the NG group (36.7\%) and SMG 10 day group (35.1\%; Fig. 3), confirming that a shorter period of SMG induces MSCs to differentiate into neuronal cells. ALP-positive cells in the SMG 10 day group accounted for $38.9 \pm 2.03 \%$ of cells, compared with the NG group $(34.3 \pm 1.28 \%)$ and SMG 72 h group $(9.5 \pm 1.12 \%$; Fig. 3), suggesting that an extended period of SMG induces MSCs to differentiate into osteogenic cells. vWF-positive cells in the SMG $72 \mathrm{~h}$ group accounted for $87.7 \pm 2.34 \%$ of cells, compared with the NG group $(77.5 \pm 1.74 \%)$ and SMG 10 day group (12.4 $\pm 1.16 \%$; Fig. 3), suggesting that a short period of SMG induces MSCs to differentiate into endothelial cells. PPAR $\gamma$-2-positive cells in the SMG 72 h group accounted for $39.7 \pm 2.01 \%$ of cells compared to the NG group $(36.9 \pm 1.18 \%)$ and SMG 10 day group (10.2 $\pm 1.11 \%$; Fig. 3). These results indicated shorter period of SMG resulted in the induction of MSCs to differentiate into adipogenic cells.

Effects of microgravity on RhoA activity. Total RhoA protein expression was confirmed by western blot analysis.
No significant change was observed in total RhoA protein expression between control cells cultured in NG or SMG. Activation of the small GTPase, RhoA, is required for both stress fiber formation and integrin-mediated signaling (14). In addition, RhoA has been implicated in the lineage decision of hMSCs (21). Therefore, the activation status of RhoA in cells cultured in SMG was examined. In the $72 \mathrm{~h}$ group, an $83 \pm 3 \%$ reduction in the activated GTP-bound form of RhoA was observed compared with the NG control group $(\mathrm{P}<0.05$; Fig. 4A). This was consistent with reduced stress fiber formation. As MSCs were exposed to SMG for 10 days, the level of activated RhoA increased nine-fold compared with the $72 \mathrm{~h}$ group ( $\mathrm{P}<0.05$; Fig. 4A).

Taken together, these data indicated that a high level of active RhoA is associated with osteogenic conditions and a low level with adipogenic, neuronal and endothelial conditions. To further examine the involvement of RhoA activation in the differentiation potential of rMSCs cultured in SMG, Y27632 (040012; Stemgent) was used to specifically block RhoA/rock signaling. Cells were pretreated with inhibitor and stimulated with SMG for 10 days. MSCs were subsequently cultured in adipogenic medium or osteogenic medium separately. The 

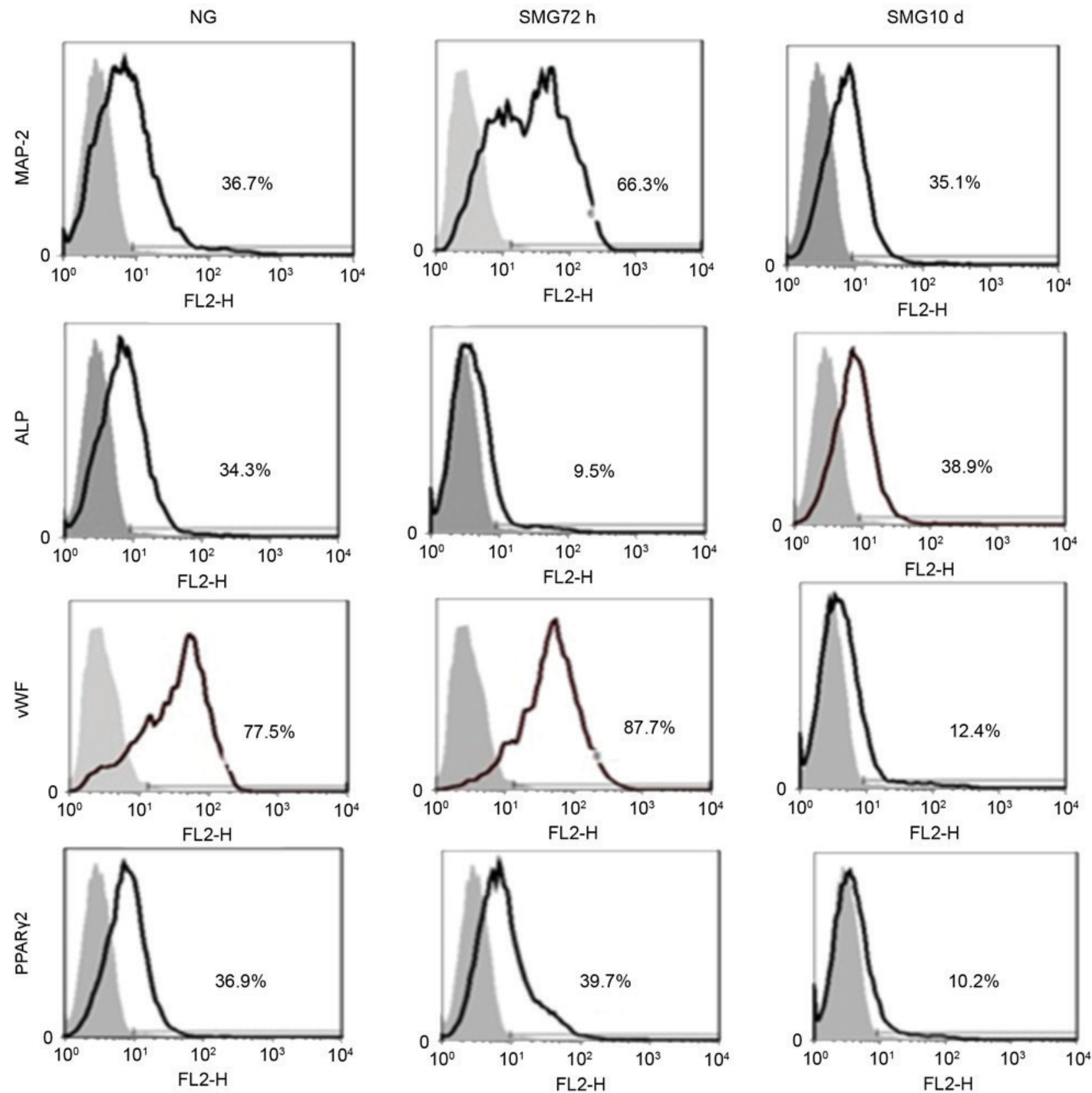

Figure 3. Fluorescence-activated cell sorting analysis of MAP-2, vWF, ALP and PPAR $\gamma-2$ following culture of rat mesenchymal stem cells for 10 days in differentiation medium subsequent to exposure to NG and $72 \mathrm{~h}$ and 10 days SMG. The gray area is the isotype control. The results represent three independent experiments. MAP-2, microtubule-associated protein 2; vWF, von Willebrand factor; ALP, alkaline phosphatase; PPAR $\gamma$ 2, peroxisome proliferator-activated receptor $\gamma 2$; NG, normal gravity; SMG, simulated microgravity.

inhibitor group was demonstrated to abrogate osteogenic media-induced osteogenesis, and redirected cells to differentiate into adipogenic cells. The expression of ALP decreased significantly by $80 \%$ in inhibited cells compared with the untreated control $(\mathrm{P}<0.05$; Fig. 4B and C). Finally, PPAR $\gamma-2$ expression increased $\sim 3$ times in the inhibited group compared with the untreated control group $(\mathrm{P}<0.05$; Fig. $4 \mathrm{~B}$ and $\mathrm{C})$.

\section{Discussion}

Bone marrow mesenchymal stem cells have demonstrated potential in tissue engineering and regenerative medicine (22). However, multiple issues still remain to be solved, including stem cell differentiation efficiency, acquiring sufficient seed cells and generating sufficient numbers of transplantation stem cells. Over the past 40 years, space exploration has revealed that microgravity has a major impact on biology. Previous studies have demonstrated that microgravity suppresses bone marrow mesenchymal stem cell differentiation toward osteoblasts, but promotes adipogenic differentiation $(8,23)$. Contrary to those findings, Buravkova et al (24) demonstrated that culture of hMSCs in microgravity for 10 days promoted osteogenic differentiation. Therefore, the effect of microgravity on stem cells is a controversial topic that requires further research attention.

The present study revealed that different durations of SMG had different effects on the cytoskeleton. Short term $72 \mathrm{~h} \mathrm{SMG}$ decreased cytoskeletal tension and resulted in microtubule 
A

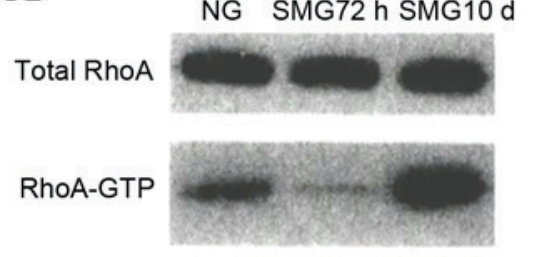

B



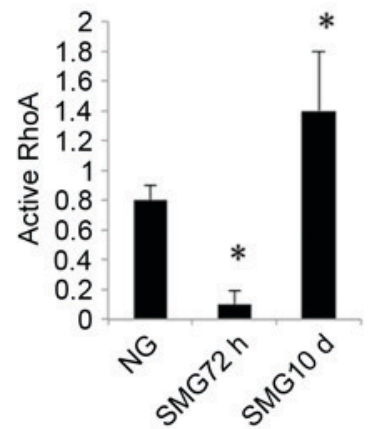

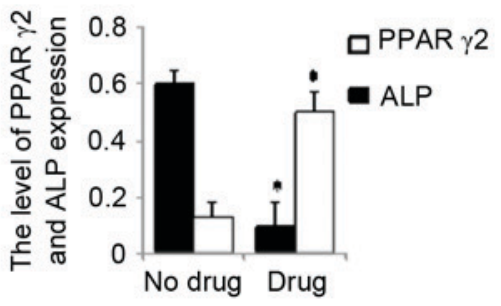

C



b

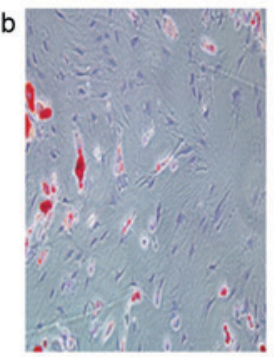

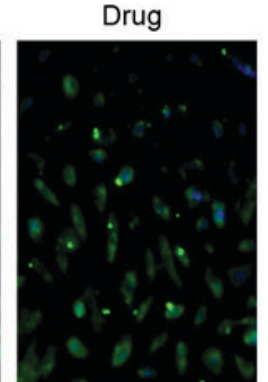



Figure 4. (A) Changes in RhoA activity following exposure to SMG for $72 \mathrm{~h}$ and 10 days. The image is representative of two independent experiments, each performed in duplicate. The band intensities of RhoA-GTP in SMG are plotted as a percentage relative to gravity controls. $\mathrm{P}<0.05$ vs. NG group. (B) PPAR $\gamma 2$ and ALP mRNA expression levels measured by semi quantitative reverse transcription polymerase chain reaction following 10 days SMG and inhibition of RhoA signaling. " $\mathrm{P}<0.05$ vs. no drug group. (C) (a) Osteopontin expression was detected by fluorescence immunocytochemistry to examine the efficiency of osteogenesis and (b) Oil Red O staining was used to examine the efficiency of adipogenic induction. The inhibitor drug group abrogated osteogenic media-induced osteogenesis, and redirected cells to the adipogenic differentiation program. RhoA, ras homolog family member A; SMG, simulated microgravity; GTP, guanosine triphosphate; NG, normal gravity; PPAR $\gamma 2$, peroxisome proliferator-activated receptor $\gamma 2$; ALP, alkaline phosphatase.

disintegration. In comparison, longer term SMG for 10 days increased cytoskeletal tension and tubulin maintained polymerization activity. Given that RhoA is involved in the regulation of the cytoskeleton, RhoA expression and activity were analyzed. Short term SMG decreased RhoA activity significantly, however, prolonged microgravity for 10 days significantly increased RhoA activity.

The effect of microgravity on bone marrow mesenchymal stem cell differentiation was also examined. A shorter period of SMG promoted MSCs to differentiate into endothelial, neuronal and adipogenic cells. In comparison, a longer period of SMG promoted MSCs to differentiate into osteoblasts. When RhoA activity was inhibited, the results revealed that the effect of longer SMG exposure was reversed. These findings highlight a clear distinction of variable microgravity duration driving bone marrow mesenchymal stem cell differentiation to different fates; one to stress-sensitive identities (including osteoblastic cells and myocardial cells), and the other to cells not sensitive to stress (including endothelial, neuronal and adipogenic cells). It has previously been demonstrated that microgravity inhibits the differentiation of osteoblasts from bone marrow mesenchymal stem cells (8). However, this is contradictory to the results of the present study, which revealed that a shorter period of SMG inhibits osteoblast differentiation, and a longer period of SMG supports osteoblast differentiation.

Spiegelman and Ginty (21) reported that changes in cell shape modulated the differentiation degree and direction. McBeath et al (25) demonstrated that cytoskeletal tension regulated stem cell differentiation fate. RhoA is an important molecule in cytoskeletal regulation, and has been reported to promote smooth and skeletal muscle cell division (26).
Sordella et al (27) demonstrated that, following elimination of RhoA by inactivating the locus, the differentiation ability of embryonic fibroblast cells into osteoblasts was enhanced, but adipogenic differentiation was weakened. These findings are consistent with the results of the present study.

RhoA is involved in cell stress fiber assembly. The most critical downstream effectors are Rho-associated, coiled-coil-containing protein kinase (ROCK) and mammalian diaphanous related protein (mDia), which are involved in the process of stress assembly (28). ROCK phosphorylates LIM domain kinase (LIMK) (29). LIMK, in turn, phosphorylates cofilin, a small actin-binding protein that promotes F-actin depolymerization. Upon phosphorylation, the actin-binding ability of cofilin is reduced, and actin polymerization is stabilized (29). mDia supports parallel stress fibers to help accumulate actin. It is also involved in the mediation of microtubule assembly and dynamic balance (30). Therefore, the effect of microgravity on cytoskeletal tension was speculated to be via the RhoA-associated pathway. A short period of SMG, which downregulates cytoskeletal tension, may inhibit RhoA to promote differentiation into cells not sensitive to stress. In comparison, a longer period of SMG upregulates cytoskeletal tension and activates RhoA to enhance stress-sensitive cell differentiation. Therefore, different periods of SMG stimulation may be an important factor that drives differentiation fates of MSCs.

In conclusion, microgravity is an important factor that affects the differentiation of rBMSCs. The findings of the present study underscore the previously reported potential of MSCs for regenerative medicine, tissue engineering and stem cell-based therapy. Future in vivo studies are required 
to confirm and clarify these findings, given that these results were obtained via in vitro simulated experiments.

\section{Acknowledgements}

The authors would like to thank Dr Austin Cape at ASJ Editors for careful reading and feedback. Confocal laser scanning microscopy was performed at the Department of Anatomy and K.K. Leung Brain Research Center, The Fourth Military Medical University (Xi'an, China). The present study was funded by the National Natural Science Foundation of China (grant no. 81402055), Natural Science Foundation of Shaanxi Province (grant no. 2016JM8014).

\section{References}

1. Langer R and Vacanti JP: Tissue engineering. Science 260 : 920-926, 1993.

2. Grimm D, Bauer J, Kossmehl P, Shakibaei M, Schöberger J, Pickenhahn H, Schulze-Tanzil G, Vetter R, Eilles C, Paul M and Cogoli A: Simulated microgravity alters differentiation and increases apoptosis in human follicular thyroid carcinoma cells. FASEB J 16: 604-606, 2002.

3. Hochleitner B, Hengster P, Duo L, Bucher H, Klima G and Margreiter R: A novel bioartificial liver with culture of porcine hepatocyte aggregates under simulated microgravity. Artif Organs 29: 58-66, 2005.

4. Marquette ML, Byerly D and Sognier M: A novel in vitro three-dimensional skeletal muscle model. In Vitro Cell Dev Biol Anim 43: 255-263, 2007.

5. Yuge L, Kajiume T, Tahara H, Kawahara Y, Umeda C, Yoshimoto R, Wu SL, Yamaoka K, Asashima M, Kataoka K and Ide T: Microgravity potentiates stem cell proliferation while sustaining the capability of differentiation. Stem Cells Dev 15: 921-929, 2006.

6. Huang Y, Dai ZQ, Ling SK, Zhang HY, Wan YM and Li YH: Gravity, a regulation factor in the differentiation of rat bone marrow mesenchymal stem cells. J Biomed Sci 16: 87, 2009.

7. Chen J, Liu R, Yang Y, Li J, Zhang X, Wang Z and Ma J: The simulated microgravity enhances the differentiation of mesenchymal stem cells into neurons. Neurosci Lett 505: 171-175, 2011

8. Dai ZQ, Wang R, Ling SK, Wan YM and Li YH: Simulated microgravity inhibits the proliferation and osteogenesis of rat bone marrow mesenchymal stem cells. Cell Prolif 40: 671-684, 2007.

9. McBeath R, Pirone DM, Nelson CM, Bhadriraju K and Chen CS: Cell shape, cytoskeletal tension, and RhoA regulate stem cell lineage commitment. Dev Cell 6: 483-495, 2004.

10. Kilian KA, Bugarija B, Lahn BT and Mrksich M: Geometric cues for directing the differentiation of mesenchymal stem cells. Proc Natl Acad Sci USA 107: 4872-4877, 2010.

11. Hughes-Fulford M and Lewis ML: Effects of microgravity on osteoblast growth activation. Exp Cell Res 224: 103-109, 1996.

12. Schatten H, Lewis ML and Chakrabarti A: Spaceflight and clinorotation cause cytoskeleton and mitochondria changes and increases in apoptosis in cultured cells. Acta Astronaut 49. 399-418, 2001.
13. Uva BM, Masini MA, Sturla M, Prato P, Passalacqua M, Giuliani M, Tagliafierro G and Strollo F: Clinorotation-induced weightlessness influences the cytoskeleton of glial cells in culture. Brain Res 934: 132-139, 2002.

14. Hall A: Rho GTPases and the actin cytoskeleton. Science 279: 509-514, 1998.

15. Azizi SA, Stokes D, Augelli BJ, DiGirolamo C and Prockop DJ: Engraftment and migration of human bone marrow stromal cells implanted in the brains of albino rats-similarities to astrocyte grafts. Proc Natl Acad Sci USA 95: 3908-3913, 1998.

16. Wang X, Ren Q, Fu S and Jiang P: Development and application of clinostat for simulation of microgravity biological effects. Acta Biophysica Sinica 13: 161-166, 1997.

17. Jiang J, Lv Z, Gu Y, Li J, Xu L, Xu W, Lu J and Xu J: Adult rat mesenchymal stem cells differentiate into neuronal-like phenotype and express a variety of neuro-regulatory molecules in vitro. Neurosci Res 66: 46-52, 2010.

18. Jaiswal N, Haynesworth SE, Caplan AI and Bruder SP: Osteogenic differentiation of purified, culture-expanded human mesenchymal stem cells in vitro. J Cell Biochem 64: 295-312, 1997.

19. Oswald J, Boxberger S, Jorgensen B, Feldmann S, Ehninger G, Bornhäuser $M$ and Werner $C$ : Mesenchymal stem cells can be differentiated into endothelial cells in vitro. Stem Cells 22: 377-384, 2004

20. Xiang Y, Zheng Q, Jia B, Huang G, Xie C, Pan J and Wang J: Ex vivo expansion, adipogenesis and neurogenesis of cryopreserved human bone marrow mesenchymal stem cells. Cell Biol Int 31: 444-450, 2007

21. Spiegelman BM and Ginty CA: Fibronectin modulation of cell shape and lipogenic gene expression in 3T3-adipocytes. Cell 35: 657-666, 1983.

22. Baksh D, Song L and Tuan RS: Adult mesenchymal stem cells: Characterization, differentiation, and application in cell and gene therapy. J Cell Mol Med 8: 301-316, 2004.

23. Zayzafoon M, Gathings WE and McDonald JM: Modeled microgravity inhibits osteogenic differentiation of human mesenchymal stem cells and increases adipogenesis. Endocrinology 145: 2421-2432, 2004.

24. Buravkova L, Romanov Y, Rykova M, Grigorieva O and Merzlikina N: Cell-to-cell interactions in changed gravity: Ground-based and flight experiments. Acta Astronaut 57: 67-74, 2005.

25. McBeath R, Pirone DM, Nelson CM, Bhadriraju K and Chen CS: Cell shape, cytoskeletal tension, and rhoa regulate stem cell lineage commitment. Dev Cell 6: 483-495, 2004.

26. Carnac G, Primig M, Kitzmann M, Chafey P, Tuil D, Lamb N and Fernandez A: RhoA GTPase and serum response factor control selectively the expression of MyoD without affecting Myf5 in mouse myoblasts. Mol Biol Cell 9: 1891-1902, 1998.

27. Sordella R, Jiang W, Chen GC, Curto M and Settleman J: Modulation of Rho GTPase signaling regulates a switch between adipogenesis and myogenesis. Cell 113: 147-158, 2003.

28. Young KG and Copeland JW: Formins in cell signaling. Biochim Biophys Acta 1803: 183-190, 2010

29. Maekawa M, Ishizaki T, Boku S, Watanabe N, Fujita A, Iwamatsu A, Obinata T, Ohashi K, Mizuno K and Narumiya S: Signaling from Rho to the actin cytoskeleton through protein kinases ROCK and LIM-kinase. Science 285: 895-898, 1999.

30. Hotulainen P and Lappalainen P: Stress fibers are generated by two distinct actin assembly mechanisms in motile cells. J Cell Biol 173: 383-394, 2006. 